\title{
Formation of hybrid block method of higher step-sizes, through the continuous multi-step collocation
}

\author{
${ }^{1}$ E. A. Ibijola, ${ }^{2}$ Y. Skwame and ${ }^{3}$ G. Kumleng \\ ${ }^{1}$ Department of Mathematical sciences, University of Ado-Ekiti , Ekiti State, Nigeria \\ ${ }^{2}$ Department of Mathematical sciences, Adamawa State University, Mubi-Nigeria. \\ ${ }^{3}$ Department of Mathematical science, University of Jos, Nigeria.
}

\begin{abstract}
We construct a self-starting Simpson's type block hybrid method (BHM) consisting of very closely accurate members each of order $\mathrm{p}=\mathrm{q}+2$ as a block. The higher order members of each were obtained by increasing the number $\mathrm{k}$ in the multi-step collocation (MC) used to derive the k-step continuous formula $(k \geq 2)$ through the aid of MAPLE soft wire program. The stability analysis is poorer as the step size $\mathrm{k}$ increases, as expected with the linear multi-step methods (Imms), discrete or continuous. In this paper we identify a continuous hybrid block schemes (CHBS) through the addition of one off-mesh collocation points in the MC. The (CHBS) is evaluated along with it's first derivative where necessary to give continuous hybrid block schemes for a simultaneous application to the stiff ordinary differential equations (SODEs\} with initial, boundary or mixed conditions.
\end{abstract}

Keywords: Continuous hybrid block schemes (CHBS), Multi-step collocation (MC), Stiff ODEs.

\section{INTRODUCTION}

The hybrid schemes have been developed since the 1960's but these methods have not as yet received a great deal of attention (see Lambert [2]) in the literature as deserve despite their higher accuracy over the single linear multi-step methods (Imms) of the same step size $\mathrm{k}$. Maybe the main reason for this may be due to the fact that, the need for special predictors to estimate the off-step solutions present in the corrector formulae.

Following Onumanyi et-al [C5] we identify a continuous hybrid scheme (CHS) through the addition of one or more off-mesh collocation points in the multi-step collocation (MC) of the form given by equation 2.18 in next section. The single (CHS) is evaluated at some distinct points involving mesh and off-mesh points along with it's first derivative, where necessary, to give multiple hybrid block schemes for the treatment of stiff ordinary differential equations.

This paper is partitioned into sections as follows. In section 2.0 we restate the MC procedure involving off-mesh collocation points for each $\mathrm{k} \geq 2$ and we analyze on its convergence analysis obtained in a block form. We obtained the order and error constants in a block form, the stability regions are also plotted. Section 3.0 is the numerical implementation of the block hybrid schemes on stiff (ODEs) and we give conclusion in section 4.0.

The method

Derivation techniques of MC.

Let us consider the first order system of ODEs

$y^{1}=f(x, y), \quad a<x<b, \quad y, f \in \mathfrak{R}^{s}$ 2.11

where $y$ satisfies a given set of $s$ associated conditions, which are either all initial, all boundary or mixed conditions. The idea of the $\mathrm{k}$ - step MC, following Onumanyi et - al $\{4\}$, is to find a polynomial $\mathrm{U}$ of the form

$$
U(x)=\sum_{j=0}^{t-1} \phi_{j}(x) y_{n+j}+h \sum_{j=0}^{m-1} \varphi_{j}(x) f\left(x_{j}, u\left(\bar{x}_{j}\right)\right), \quad x_{n} \leq x \leq x_{n+x}
$$

Where $t$ denotes the number of interpolation points $x_{n+i}, \quad i=0,1,---, t-1$, and $m$ denote the number of distinct collocation points $\overline{x_{i}} \in\left[x_{n}, x_{n+k}\right], \quad i=0,1,---, m-1$ the point $s \overline{x_{i}}$ are chosen from the step $x_{n+i}$ as well as one or more off - step points.

The following assumptions are made;

1. Although the step size can be variable, for simplicity in our presentation of the analysis in this paper, we assume it is constant 
$h=x_{n+1}-x_{n}, \quad N=\frac{b-a}{h}$ with the steps given by $\left\{x_{n} / x_{n}=a+n h, n=0,1,---, N\right\}$,

2. That (2.11) has a unique solution and the coefficients $\phi_{j}(x), \varphi_{j}(x)$ in (2.12) can be represented by polynomials of the form

$\phi_{j}(x)=\sum_{i=0}^{t+m-1} \phi_{j, i+1} x^{i}, \quad j \varepsilon\{0,1,---, t-1\} \quad-----2.13$

$\mathrm{h} \varphi_{j}(x)=\sum_{i=0}^{t+m-1} \varphi_{j, i+1} x^{i} \quad j \varepsilon\{0,1,---, m-1\}$

With constant coefficients $\phi_{j, i+1}, h \varphi_{j, i+1}$ to be determined using the interpolation and collocation conditions:

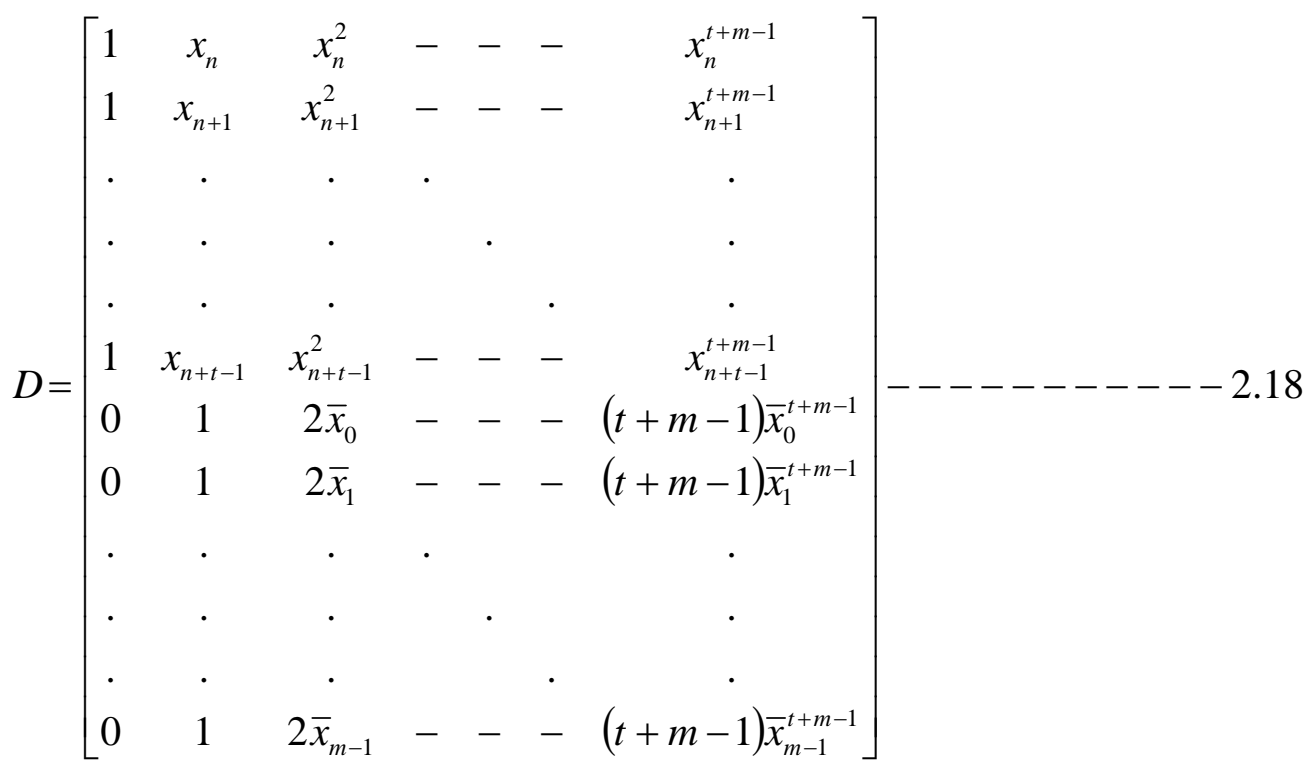

$$
\begin{aligned}
& u\left(x_{n+i}\right)=y_{n+i}, i \varepsilon\{0,1,---, t-1\}----2.15 \\
& u^{1}\left(x_{i}\right)=f\left(x_{i}, u\left(x_{j}\right)\right), j \varepsilon\{0,1,-m-1\}
\end{aligned}
$$

With this assumptions we obtain an MC polynomial, following [C 5], in the form

$$
u(x)=\sum_{i=0}^{t+m-1} a_{i} x^{i}, \quad a_{i}=\sum_{j=0}^{t-1} c_{i+1, j+1}+\sum_{j=0}^{m-1} c_{i+1, j+t+1} f_{n+j}
$$

Where $x_{n} \leq x \leq x_{n+k}$ and $c_{i j} i, j \varepsilon\{1,2,---, t+m\}$ are constants given by the elements of the inverse matrix $C=D^{-1}$. The MC matrix $\mathrm{D}$ is a nonsingular $(m+1)$ square matrix of the type

The parameters required for equation (2.18) are $\mathrm{k}=2, \mathrm{t}=1, \mathrm{~m}=\mathrm{k}+2 ;\left(x_{n}, x_{n+1}\right)$ :

$\bar{x}_{0}=x_{n}, \quad \bar{x}_{1}=x_{n+1}, \quad \bar{x}_{\frac{3}{2}}=x_{n+\frac{3}{2}},---$

The matrix (2.18) takes the following shape

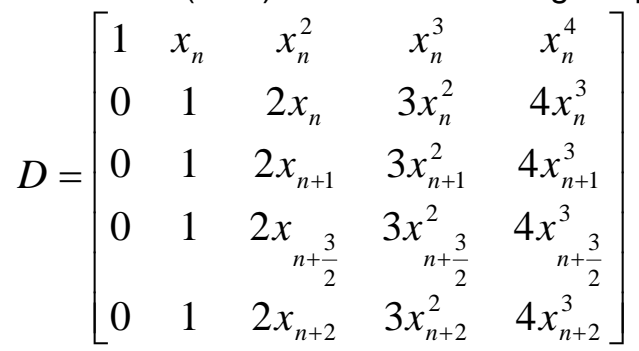

By using the maple software program and evaluating (2.19) at the grid points 
$x=x_{n+1} ; x=x_{n+\frac{3}{2}} ; x=x_{n+2}$ we obtain the three discrete schemes, interesting the integrator obtained at $x=x_{n+2}$, is the most popular Simpson's $\frac{1}{3}$ rule hence, the schemes are

$$
\begin{gathered}
y_{n+1}=y\left(x=x_{n+1}\right)=y_{n}+\frac{h}{6}\left[2 f_{n}+7 f_{n+1}-4 f_{n+\frac{3}{2}}+f_{n+2}\right] \\
y_{n+\frac{3}{2}}=y\left(x=x_{n+\frac{3}{2}}\right)=y_{n}+\frac{3 h}{64}\left[7 f_{n}+30 f_{n+1}-8 f_{n+\frac{3}{2}}+3 f_{n+2}\right] \\
y_{n+2}=y\left(x=x_{n+2}\right)=y_{n}+\frac{h}{3}\left[f_{n}+4 f_{n+1}+f_{n+2}\right]
\end{gathered}
$$

Therefore the hybrid block methods are

$$
\begin{aligned}
& y_{n+1}=y_{n}+\frac{h}{6}\left[2 f_{n}+7 f_{n+1}-4 f_{n+\frac{3}{2}}+f_{n+2}\right] \\
& y_{n+\frac{3}{2}}=y_{n}+\frac{3 h}{64}\left[7 f_{n}+30 f_{n+1}-8 f_{n+\frac{3}{2}}+3 f_{n+2}\right]----2.20 \\
& y_{n+2}=y_{n}+\frac{h}{3}\left[f_{n}+4 f_{n+1}+f_{n+2}\right]
\end{aligned}
$$

Also, the parameters required for equation (2.18) are $\mathrm{k}=3, \mathrm{t}=1, \mathrm{~m}=\mathrm{k}+2 ;\left(x_{n}, x_{n+1}\right)$;

$$
\left(\overline{x_{0}}=x_{n}, \overline{x_{1}}=x_{n+1}, \overline{x_{2}}=x_{n+2}, \overline{x_{\frac{5}{2}}}=x_{n+\frac{5}{2}}, \overline{x_{3}}=x_{n+3}\right)
$$

Hence the matrix (2.18) takes the following shape

$$
D=\left[\begin{array}{cccccc}
1 & x_{n} & x_{n}^{2} & x_{n}^{3} & x_{n}^{4} & x_{n}^{5} \\
0 & 1 & 2 x_{n} & 3 x_{n}^{2} & 4 x_{n}^{3} & 5 x_{n}^{4} \\
0 & 1 & 2 x_{n+1} & 3 x_{n+1}^{2} & 4 x_{n+1}^{3} & 5 x_{n+1}^{4} \\
0 & 1 & 2 x_{n+2} & 3 x_{n+2}^{2} & 4 x_{n+2}^{3} & 5 x_{n+2}^{4} \\
0 & 1 & 2 x_{n+\frac{5}{2}} & 3 x^{2} & 4 x^{3} & 5 x^{4} \\
0 & 1 & 2 x_{n+3} & 3 x_{n+3}^{2} & 4 x_{n+3}^{3} & 5 x_{n+3}^{4}
\end{array}\right]
$$

Using the maple soft ware environment to evaluate (2.21) at the grid points

$$
x=x_{n+1}, x=x_{n+2}, x=x_{n+\frac{5}{2}}, x=x_{n+3}
$$

We obtain the four discrete schemes, namely,

$$
y_{n+1}=y_{n}+h\left[\frac{599}{1800} f_{n}+\frac{361}{360} f_{n+1}-\frac{101}{120} f_{n+2}+\frac{152}{225} f_{n+\frac{5}{2}}-\frac{61}{360} f_{n+3}\right]
$$




$$
\begin{aligned}
& y_{n+2}=y_{n}+h\left[\frac{71}{225} f_{n}+\frac{64}{45} f_{n+1}+\frac{1}{15} f_{n+2}+\frac{64}{225} f_{n+\frac{5}{2}}-\frac{4}{45} f_{n+3}\right] \\
& y_{n+\frac{5}{2}}=y_{n}+h\left[\frac{365}{1152} f_{n}+\frac{1625}{1152} f_{n+1}+\frac{125}{384} f_{n+2}+\frac{5}{9} f_{n+\frac{5}{2}}-\frac{125}{1152} f_{n+3}\right] \\
& y_{n+3}=y_{n}+h\left[\frac{63}{200} f_{n}+\frac{57}{40} f_{n+1}+\frac{9}{40} f_{n+2}+\frac{24}{25} f_{n+\frac{5}{2}}+\frac{3}{40} f_{n+3}\right]
\end{aligned}
$$

We follow the same procedure to obtain the hybrid block method for $k=4$ from equation (2.18) as

$$
\begin{aligned}
& y_{n+1}=y_{n}+h\left[\frac{811}{2520} f_{n}+\frac{377}{360} f_{n+1}-\frac{89}{120} f_{n+2}+\frac{323}{360} f_{n+3}-\frac{24}{35} f_{n+\frac{7}{2}}+\frac{29}{180} f_{n+4}\right] \\
& y_{n+2}=y_{n}+h\left[\frac{193}{630} f_{n}+\frac{22}{15} f_{n+1}+\frac{2}{45} f_{n+2}+\frac{22}{45} f_{n+3}-\frac{128}{315} f_{n+\frac{7}{2}}+\frac{1}{10} f_{n+4}\right] \\
& y_{n+3}=y_{n}+h\left[\frac{87}{280} f_{n}+\frac{57}{40} f_{n+1}+\frac{21}{40} f_{n+2}+\frac{51}{40} f_{n+3}-\frac{24}{35} f_{n+\frac{7}{2}}+\frac{3}{20} f_{n+4}\right] \\
& y_{n+\frac{7}{2}}=y_{n}+h\left[\frac{7147}{23040} f_{n}+\frac{343}{240} f_{n+1}+\frac{5831}{11520} f_{n+2}+\frac{4459}{2880} f_{n+3}-\frac{77}{180} f_{n+\frac{7}{2}}+\frac{243}{2560} f_{n+4}\right] \\
& y_{n+4}=y_{n}+h\left[\frac{14}{45} f_{n}+\frac{64}{45} f_{n+1}+\frac{8}{15} f_{n+2}+\frac{64}{45} f_{n+3}+\frac{14}{45} f_{n+4}\right]
\end{aligned}
$$

\section{Stability of Block Method}

The equations 2.20 when put together formed the block as

$$
\left[\begin{array}{lll}
1 & 0 & 0 \\
0 & 1 & 0 \\
0 & 0 & 1
\end{array}\right]\left[\begin{array}{c}
y_{n+1} \\
y_{n+\frac{3}{2}} \\
y_{n+2}
\end{array}\right]=\left[\begin{array}{lll}
0 & 0 & 1 \\
0 & 0 & 1 \\
0 & 0 & 1
\end{array}\right]\left[\begin{array}{c}
y_{n+2} \\
y_{n+1} \\
y_{n}
\end{array}\right]+h\left[\begin{array}{ccc}
\frac{7}{6} & -\frac{2}{3} & \frac{1}{6} \\
\frac{90}{64} & -\frac{24}{64} & \frac{9}{64} \\
\frac{4}{3} & 0 & \frac{1}{3}
\end{array}\right]\left[\begin{array}{c}
f_{n+1} \\
f_{n+\frac{3}{2}} \\
f_{n+2}
\end{array}\right]+h\left[\begin{array}{ccc}
0 & 0 & \frac{1}{3} \\
0 & 0 & \frac{24}{64} \\
0 & 0 & \frac{1}{3}
\end{array}\right]\left[\begin{array}{c}
f_{n+2} \\
f_{n+\frac{3}{2}} \\
f_{n}
\end{array}\right]----2.31 \text { Nor }
$$

malizing (2.31) by multiplying with the inverse of $A^{(0)}$ we obtained:

$$
\left[\begin{array}{lll}
1 & 0 & 0 \\
0 & 1 & 0 \\
0 & 0 & 1
\end{array}\right]\left[\begin{array}{c}
y_{n+1} \\
y_{n+\frac{3}{2}} \\
y_{n+2}
\end{array}\right]=\left[\begin{array}{lll}
0 & 0 & 1 \\
0 & 0 & 1 \\
0 & 0 & 1
\end{array}\right]\left[\begin{array}{c}
y_{n+2} \\
y_{n+1} \\
y_{n}
\end{array}\right]+h\left[\begin{array}{ccc}
\frac{7}{6} & -\frac{2}{3} & \frac{1}{6} \\
\frac{90}{64} & -\frac{24}{64} & \frac{9}{64} \\
\frac{4}{3} & 0 & \frac{1}{3}
\end{array}\right]\left[\begin{array}{c}
f_{n+1} \\
f_{n+\frac{3}{2}} \\
f_{n+2}
\end{array}\right]+h\left[\begin{array}{ccc}
0 & 0 & \frac{1}{3} \\
0 & 0 & \frac{24}{64} \\
0 & 0 & \frac{1}{3}
\end{array}\right]\left[\begin{array}{c}
f_{n+2} \\
f_{n+\frac{3}{2}} \\
f_{n}
\end{array}\right]----2.32 \text { The first }
$$

characteristic polynomial of the hybrid block method (2.17) and (2.32) is given as 


$$
\begin{aligned}
\rho(R) & =\operatorname{det}\left[R A^{0}-A^{1}\right] \text { where } A^{0}=\left[\begin{array}{lll}
1 & 0 & 0 \\
0 & 1 & 0 \\
0 & 0 & 1
\end{array}\right] \text { and } A^{1}=\left[\begin{array}{lll}
0 & 0 & 1 \\
0 & 0 & 1 \\
0 & 0 & 1
\end{array}\right] \\
\rho(R) & =\operatorname{det}\left[R\left(\begin{array}{lll}
1 & 0 & 0 \\
0 & 1 & 0 \\
0 & 0 & 1
\end{array}\right)-\left(\begin{array}{lll}
0 & 0 & 1 \\
0 & 0 & 1 \\
0 & 0 & 1
\end{array}\right)\right] \\
& =\operatorname{det}\left[\left(\begin{array}{lll}
R & 0 & 0 \\
0 & R & 0 \\
0 & 0 & R
\end{array}\right)-\left(\begin{array}{lll}
0 & 0 & 1 \\
0 & 0 & 1 \\
0 & 0 & 1
\end{array}\right)\right] \\
& =\operatorname{det}\left[\begin{array}{lll}
R & 0 & -1 \\
0 & R & -1 \\
0 & 0 & R-1
\end{array}\right] \\
& =R(R(R-1)) \Rightarrow R_{1}=0, R_{2}=0, R_{3}=1
\end{aligned}
$$

Since $\left|R_{j}\right| \leq 1, j \varepsilon\{1,2,3\}$ hence the method as a block is zero stable on its own. The hybrid block method is also consistent as its order $\mathrm{p}>1$.

From Henrici (1962), we can safely assert the convergence of the hybrid block method (2.31)

\section{Convergence Analysis.}

\section{Order and Error constants of the Block Hybrid Methods.}

The hybrid block methods which are obtained in a block form with the help of a maple soft ware have the following order and error constants for each case.

\section{Case $k=2$}

$\begin{array}{lcc}\text { Evaluating points } & \text { Order } & \text { Error constants } \\ y\left(x=x_{n+1}\right) & 4 & -\frac{31}{2880} \\ y\left(x=x_{n+\frac{3}{2}}\right) & 4 & -\frac{51}{5120} \\ y\left(x=x_{n+2}\right) & 4 & -\frac{1}{90}\end{array}$




\section{Case $k=3$}

Evaluating points

$y\left(x=x_{n+1}\right)$

$y\left(x=x_{n+2}\right)$

$y\left(x=x_{n+\frac{5}{2}}\right)$

$y\left(x=x_{n+3}\right)$
Order

5

5

5

5

Case $\mathrm{k}=4$

Evaluating points

$y\left(x=x_{n+1}\right)$

$y\left(x=x_{n+2}\right)$

$y\left(x=x_{n+3}\right)$

$y\left(x=x_{n+\frac{7}{2}}\right)$

$y\left(x=x_{n+4}\right)$
Order

6

6

6

6

6

\section{Error constants}

$\frac{13}{1200}$

$\frac{7}{900}$

$\frac{25}{3072}$

$\frac{3}{400}$

$-\frac{1159}{120960}$

$-\frac{53}{7560}$

$-\frac{37}{4480}$

$-\frac{4459}{552960}$

$-\frac{8}{945}$

Stability Region of the Hybrid Block Methods.

The stability function is given by:

$M(Z)=B_{2}+Z A_{2}\left(I-Z A_{1}\right)^{-1} B_{1} \quad--------2.51$

For the stability properties of the block method, is reformulated as a general linear method of the form:

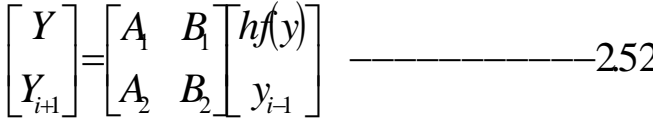

The block method is partitioned in a matrix form as: 


$$
\left[\begin{array}{c}
Y \\
y_{i+1}
\end{array}\right]=\left[\begin{array}{cc}
A & U \\
B & V
\end{array}\right]\left[\begin{array}{c}
H f(y) \\
y_{i-1}
\end{array}\right]
$$

where, $A=\left[\begin{array}{ccccc}a_{11} & . & . & . & a_{1 s} \\ \cdot & & & & \\ \cdot & & & & \\ \cdot & & & & \\ a_{s 1} & \cdot & \cdot & \cdot & a_{s s}\end{array}\right], B=\left[\begin{array}{ccccc}b_{11} & \cdot & \cdot & . & b_{1 s} \\ \cdot & & & \\ \cdot & \\ \cdot & & & & \\ b_{k 1} & \cdot & \cdot & \cdot & b_{k s}\end{array}\right], Y=\left[\begin{array}{c}y_{n} \\ y_{n+1} \\ \cdot \\ \cdot \\ \cdot \\ y_{n+k}\end{array}\right]$,

$y_{i+1}=\left[\begin{array}{c}y_{n+k} \\ \cdot \\ \cdot \\ \cdot \\ y_{n+k-1}\end{array}\right], \quad y_{i-1}=\left[\begin{array}{c}y_{n+k-1} \\ \cdot \\ \cdot \\ \cdot \\ y_{n}\end{array}\right]$

We obtain the element of the matrices $A$ and $B$ from the coefficients of the collocation points and the elements of $U$ and $V$ are obtained from the interpolation points, comparing (2.52) and (2.53), we see that $A=A_{1}, B=A_{2}, U=B_{1}, V=B_{2}$.

Given the hybrid block Simpson's method; for $k \geq 2$. From equation (2.20), we write the block method in a matrix form as (2.53).

$$
\begin{gathered}
\text { Case } \mathrm{k}=2 \\
{\left[\begin{array}{c}
y_{n} \\
y_{n+1} \\
y_{n+\frac{3}{2}} \\
y_{n+2} \\
---- \\
y_{n+2} \\
y_{n+1}
\end{array}\right]\left[\begin{array}{ccccccc}
0 & 0 & 0 & 0 & \cdot & 0 & 1 \\
\frac{2}{6} & \frac{7}{6} & -\frac{4}{6} & \frac{1}{6} & \cdot & 0 & 1 \\
\frac{21}{64} & \frac{90}{64} & -\frac{24}{64} & \frac{9}{64} & \cdot & 0 & 1 \\
\frac{1}{3} & \frac{4}{3} & 0 & \frac{1}{3} & \cdot & 0 & 1 \\
-\frac{1}{3} & -\frac{4}{3} & -- & -\frac{1}{3} & -- & -- & -- \\
\frac{2}{6} & \frac{7}{6} & -\frac{4}{6} & \frac{1}{6} & \cdot & 0 & 1
\end{array}\right]\left[\begin{array}{c}
f_{n} \\
f_{n+1} \\
f_{n+\frac{3}{2}} \\
f_{n+2} \\
-- \\
f_{n+1} \\
f_{n}
\end{array}\right]}
\end{gathered}
$$

Where, 


$$
A=A_{1}=\left[\begin{array}{cccc}
0 & 0 & 0 & 0 \\
\frac{2}{6} & \frac{7}{6} & -\frac{4}{6} & \frac{1}{6} \\
\frac{21}{64} & \frac{90}{64} & -\frac{24}{64} & \frac{9}{64} \\
\frac{1}{3} & \frac{4}{3} & 0 & \frac{1}{3}
\end{array}\right], U=B_{1}=\left[\begin{array}{ll}
0 & 1 \\
0 & 1 \\
0 & 1 \\
0 & 1
\end{array}\right], B=A_{2}=\left[\begin{array}{cccc}
\frac{1}{3} & \frac{4}{3} & 0 & \frac{1}{3} \\
\frac{2}{6} & \frac{7}{6} & -\frac{4}{6} & \frac{1}{6}
\end{array}\right], V=B_{2}=\left[\begin{array}{ll}
0 & 1 \\
0 & 1
\end{array}\right]
$$

\section{Case $k=3$}

\begin{tabular}{|c|c|c|c|c|c|c|c|c|c|c|}
\hline & $\begin{array}{c}0 \\
599\end{array}$ & $\begin{array}{c}0 \\
361\end{array}$ & $\begin{array}{l}0 \\
101\end{array}$ & $\begin{array}{c}0 \\
152\end{array}$ & $\begin{array}{l}0 \\
61\end{array}$ & . & 0 & 0 & 1 & \\
\hline$y_{n}$ & 1800 & 360 & 120 & 225 & 360 & • & 0 & 0 & 1 & $f_{n}$ \\
\hline$y_{n+1}$ & 71 & 64 & 1 & 64 & 4 & & 0 & 0 & 1 & $t_{n+1}$ \\
\hline$y_{n+2}$ & $\begin{array}{l}225 \\
365\end{array}$ & 45 & 15 & 225 & 45 & & & & & r ITT \\
\hline & 305 & $10 \angle 5$ & & & 125 & & 0 & 0 & 1 & \\
\hline$y_{n+\frac{5}{2}}$ & 1152 & 1152 & 384 & 9 & 1152 & & & & & $t_{n+-}$ \\
\hline$y_{n+3}$ & 200 & 40 & 40 & 25 & 40 & $\cdot$ & 0 & 0 & 1 & $f_{n+3}$ \\
\hline--- & --- & --- & $-\frac{-}{0}-$ & --- & $-\frac{-}{2}-$ & -- & -- & -- & -- & -- \\
\hline$y_{n+3}$ & 03 & / & 9 & 24 & 3 & • & 0 & 0 & 1 & $f_{n+2}$ \\
\hline$y_{n+2}$ & $\begin{array}{c}200 \\
71\end{array}$ & $\begin{array}{l}40 \\
64\end{array}$ & $\begin{array}{c}40 \\
1\end{array}$ & $\begin{array}{l}25 \\
64\end{array}$ & $\begin{array}{r}40 \\
4\end{array}$ & & & & & $f_{n+1}$ \\
\hline$y_{n+1}$ & 225 & 45 & 15 & 225 & 45 & ${ }^{\circ}$ & 0 & 0 & 1 & $f_{n}$ \\
\hline & 599 & 361 & 101 & 152 & & & 0 & 0 & 1 & \\
\hline & L1800 & 360 & 120 & 225 & 360 & & & & & \\
\hline
\end{tabular}

Where,

$$
A=\left[\begin{array}{ccccc}
0 & 0 & 0 & 0 & 0 \\
\frac{599}{1800} & \frac{361}{360} & -\frac{101}{120} & \frac{152}{225} & -\frac{61}{360} \\
\frac{71}{225} & \frac{64}{45} & \frac{1}{15} & \frac{64}{225} & -\frac{4}{45} \\
\frac{365}{1152} & \frac{1625}{1152} & \frac{125}{384} & \frac{5}{9} & -\frac{125}{1152} \\
\frac{63}{200} & \frac{57}{40} & \frac{9}{40} & \frac{24}{25} & \frac{3}{40}
\end{array}\right], \quad U=\left[\begin{array}{lll}
0 & 0 & 1 \\
0 & 0 & 1 \\
0 & 0 & 1 \\
0 & 0 & 1 \\
0 & 0 & 1
\end{array}\right]
$$




$$
B=\left[\begin{array}{ccccc}
\frac{63}{200} & \frac{57}{40} & \frac{9}{40} & \frac{24}{25} & \frac{3}{40} \\
\frac{71}{225} & \frac{64}{45} & \frac{1}{15} & \frac{64}{225} & -\frac{4}{45} \\
\frac{599}{1800} & \frac{361}{360} & -\frac{101}{120} & \frac{152}{225} & -\frac{61}{360}
\end{array}\right], \quad V=\left[\begin{array}{ccc}
0 & 0 & 1 \\
0 & 0 & 1 \\
0 & 0 & 1
\end{array}\right]
$$

Case $\mathrm{k}=4$

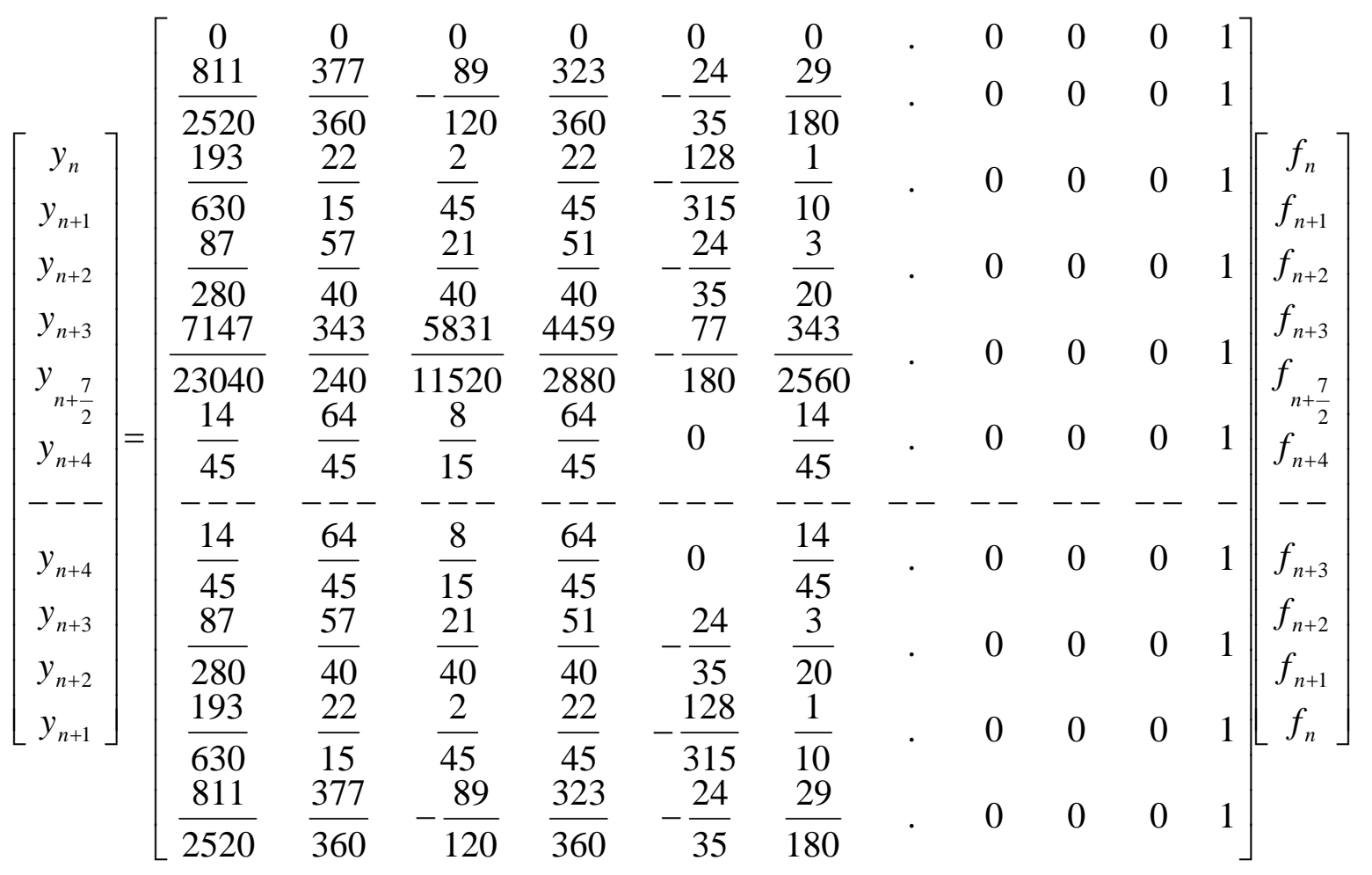

Where,

$$
A=\left[\begin{array}{cccccc}
0 & 0 & 0 & 0 & 0 & 0 \\
\frac{811}{2520} & \frac{377}{360} & -\frac{89}{120} & \frac{323}{360} & -\frac{24}{35} & \frac{29}{180} \\
\frac{193}{630} & \frac{22}{15} & \frac{2}{45} & \frac{22}{45} & -\frac{128}{315} & \frac{1}{10} \\
\frac{87}{280} & \frac{57}{40} & \frac{21}{40} & \frac{51}{40} & -\frac{24}{35} & \frac{3}{20} \\
\frac{7147}{23040} & \frac{243}{240} & \frac{5831}{11520} & \frac{4459}{2880} & -\frac{77}{180} & \frac{343}{2560} \\
\frac{14}{45} & \frac{64}{45} & \frac{8}{15} & \frac{64}{45} & 0 & \frac{14}{45}
\end{array}\right], \quad U=\left[\begin{array}{cccc}
0 & 0 & 0 & 1 \\
0 & 0 & 0 & 1 \\
0 & 0 & 0 & 1 \\
0 & 0 & 0 & 1 \\
0 & 0 & 0 & 1 \\
0 & 0 & 0 & 1
\end{array}\right]
$$


Am. J. Sci. Ind. Res., 2011, 2(2): 161-173

$$
B=\left[\begin{array}{cccccc}
\frac{14}{45} & \frac{64}{45} & \frac{8}{15} & \frac{64}{45} & 0 & \frac{14}{45} \\
\frac{87}{280} & \frac{57}{40} & \frac{21}{40} & \frac{51}{40} & -\frac{24}{35} & \frac{3}{20} \\
\frac{193}{630} & \frac{22}{15} & \frac{2}{45} & \frac{22}{45} & -\frac{128}{315} & \frac{1}{10} \\
\frac{811}{2520} & \frac{377}{360} & -\frac{89}{120} & \frac{323}{360} & -\frac{24}{35} & \frac{29}{180}
\end{array}\right], \quad V=\left[\begin{array}{llll}
0 & 0 & 0 & 1 \\
0 & 0 & 0 & 1 \\
0 & 0 & 0 & 1 \\
0 & 0 & 0 & 1
\end{array}\right]
$$

The elements of the matrices $A, B, U$ and $V$ are substituted and computing the stability function with Maple software yield, the stability polynomial of the method which is then plotted in MATLAB environment to produce the required absolute stability region of the methods, as shown by the figures $2.60,2.61$ and 2.62 .

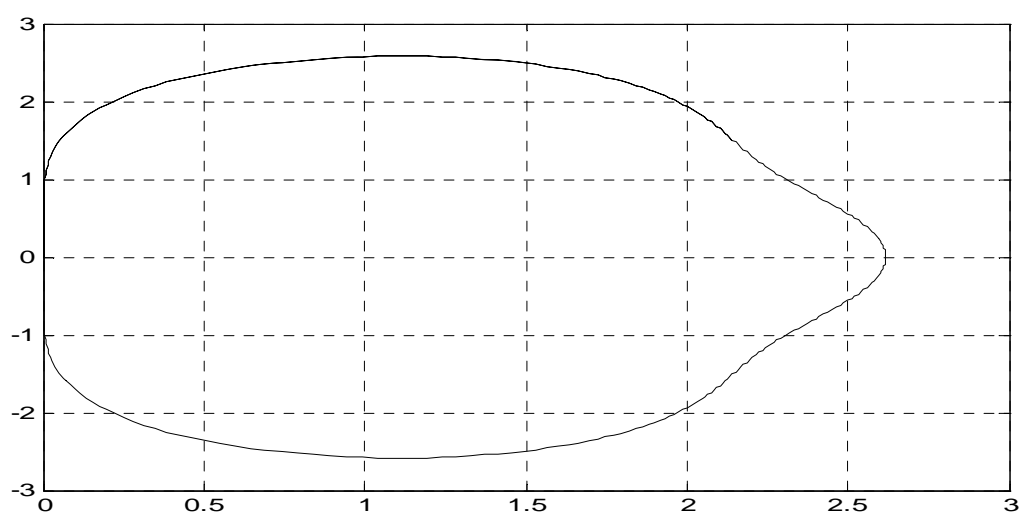

Figure 2.60: Stability region of block Simpson's method for $k=2$ with one off-mesh point.

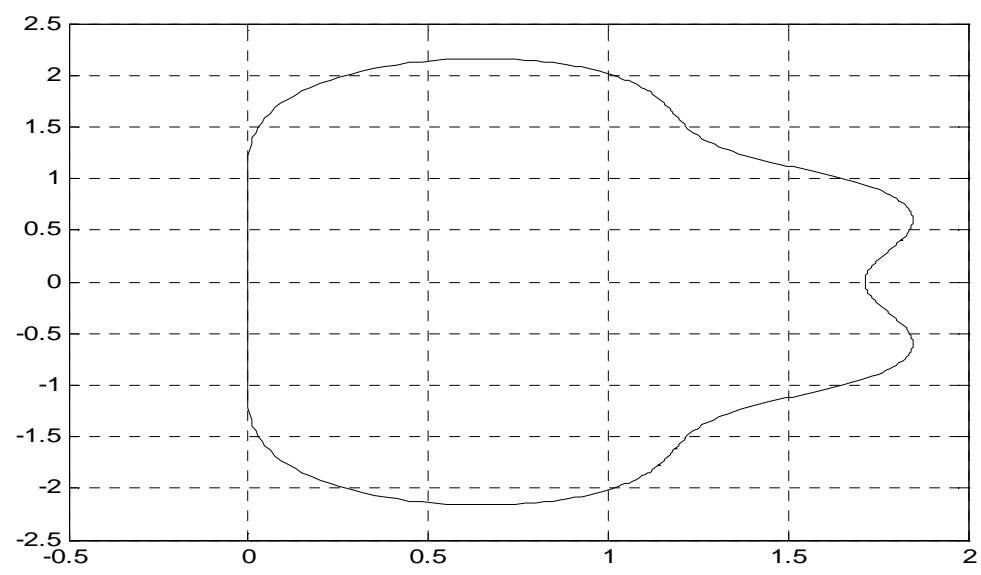

Figure 2.61: Stability region of the block hybrid Simpson's method for k=3 with one off-mesh point. 


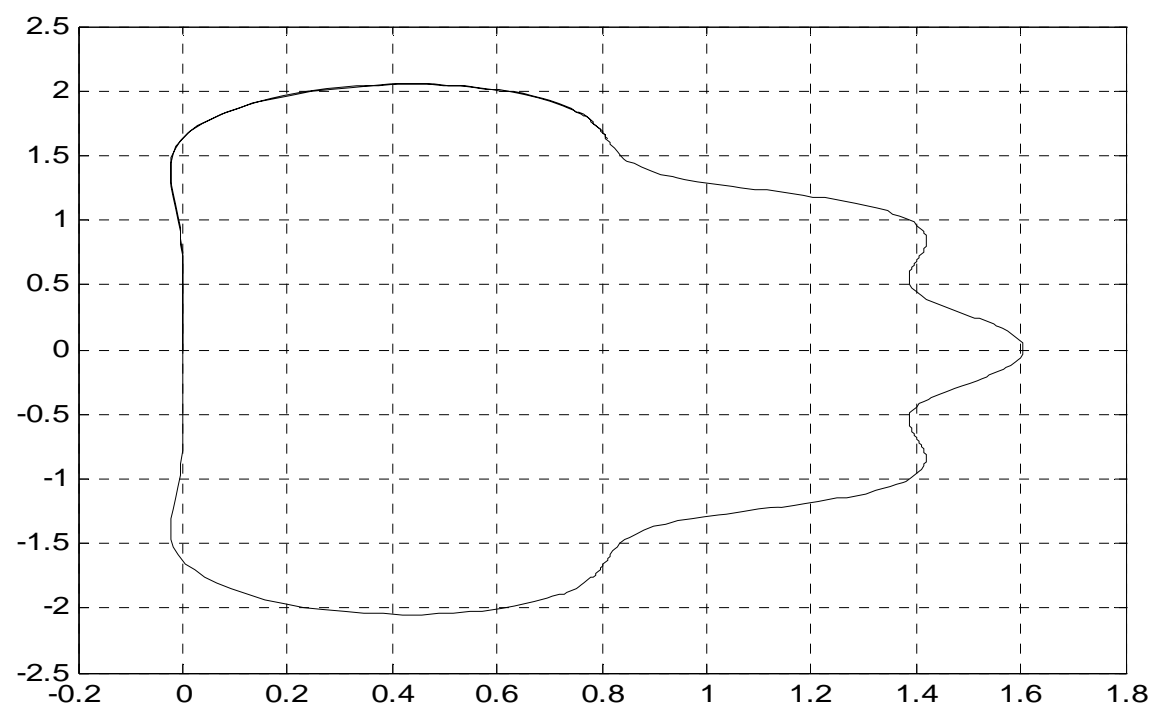

Figure 2.62: Stability region of the block hybrid Simpson's method for $k=4$ with one off-mesh point

\section{Numerical Implementation.}

To study the efficiency of the block hybrid method for $k \geq 2$, we present some numerical examples widely used by several authors such as Nakashima [8] and Yakubu [3].

Example1. $\quad y^{1}=-1,000,000 y, \quad y(0)=1, h=0.1, x \varepsilon[0,2]$,

$$
\text { Exact solution } y(x)=e^{-1,000,000 x} \text {. }
$$

Example $2 \quad y^{1}=-10 y, \quad y(0)=1, h=0.1, \quad x \varepsilon[0,2]$

$$
y(x)=e^{-10 y} \text {. }
$$


Table 1: Absolute errors of numerical solution for example 1.

\begin{tabular}{|l|l|l|l|}
\hline $\mathrm{Y}$ & $\begin{array}{l}\text { Hybrid Block Simpson's } \\
\mathrm{K}=2\end{array}$ & $\begin{array}{l}\text { Hybrid Block Simpson's } \\
\mathrm{K}=3\end{array}$ & $\begin{array}{l}\text { Hybrid Block Simpson's } \\
\mathrm{K}=4\end{array}$ \\
\hline 0.1 & $1.66 \times 10^{-1}$ & $1.99 \times 10^{-1}$ & $1.78 \times 10^{-1}$ \\
\hline 0.2 & $3.33 \times 10^{-1}$ & $6.66 \times 10^{-2}$ & $7.14 \times 10^{-2}$ \\
\hline 0.3 & $5.55 \times 10^{-2}$ & $1.99 \times 10^{-1}$ & $3.57 \times 10^{-2}$ \\
\hline 0.4 & $1.11 \times 10^{-1}$ & $3.99 \times 10^{-2}$ & $1.42 \times 10^{-1}$ \\
\hline 0.5 & $3.70 \times 10^{-2}$ & $1.33 \times 10^{-2}$ & $2.55 \times 10^{-2}$ \\
\hline 0.6 & $2.59 \times 10^{-1}$ & $3.99 \times 10^{-2}$ & $1.02 \times 10^{-2}$ \\
\hline 0.7 & $4.31 \times 10^{-2}$ & $1.59 \times 10^{-3}$ & $5.10 \times 10^{-3}$ \\
\hline 0.8 & $8.63 \times 10^{-2}$ & $5.33 \times 10^{-4}$ & $2.04 \times 10^{-2}$ \\
\hline 0.9 & $1.43 \times 10^{-2}$ & $1.59 \times 10^{-3}$ & $5.20 \times 10^{-4}$ \\
\hline 1.0 & $2.87 \times 10^{-2}$ & $2.55 \times 10^{-6}$ & $2.08 \times 10^{-4}$ \\
\hline 1.1 & $4.79 \times 10^{-3}$ & $8.52 \times 10^{-1}$ & $1.04 \times 10^{-4}$ \\
\hline 1.2 & $9.59 \times 10^{-3}$ & $2.55 \times 10^{-6}$ & $4.16 \times 10^{-4}$ \\
\hline 1.3 & $1.59 \times 10^{-3}$ & $6.54 \times 10^{-12}$ & $2.16 \times 10^{-1}$ \\
\hline 1.4 & $3.19 \times 10^{-3}$ & $2.18 \times 10^{-12}$ & $8.66 \times 10^{-8}$ \\
\hline 1.5 & $5.33 \times 10^{-4}$ & $6.54 \times 10^{-12}$ & $4.33 \times 10^{-8}$ \\
\hline 1.6 & $1.06 \times 10^{-3}$ & $4.28 \times 10^{-23}$ & $1.73 \times 10^{-1}$ \\
\hline 1.7 & $1.77 \times 10^{-4}$ & $1.42 \times 10^{-23}$ & $3.75 \times 10^{-14}$ \\
\hline 1.8 & $3.55 \times 10^{-4}$ & $4.28 \times 10^{-23}$ & $1.50 \times 10^{-14}$ \\
\hline 1.9 & $5.92 \times 10^{-5}$ & $1.83 \times 10^{-45}$ & $7.50 \times 10^{-15}$ \\
\hline 2.0 & $1.18 \times 10^{-4}$ & $6.12 \times 10^{-46}$ & $3.00 \times 10^{-14}$ \\
\hline
\end{tabular}

Table 2: Absolute errors of numerical solution for example 2.

\begin{tabular}{|l|l|l|l|}
\hline $\mathrm{Y}$ & $\begin{array}{l}\text { Hybrid Block Simpson's } \\
\mathrm{K}=2\end{array}$ & $\begin{array}{l}\text { Hybrid Block Simpson's } \\
\mathrm{K}=3\end{array}$ & $\begin{array}{l}\text { Hybrid Block Simpson's } \\
\mathrm{K}=4\end{array}$ \\
\hline 0.1 & $2.20 \times 10^{-3}$ & $1.33 \times 10^{-3}$ & $7.13 \times 10^{-4}$ \\
\hline 0.2 & $1.90 \times 10^{-3}$ & $1.03 \times 10^{-4}$ & $6.34 \times 10^{-5}$ \\
\hline 0.3 & $8.85 \times 10^{-4}$ & $3.49 \times 10^{-5}$ & $1.21 \times 10^{-4}$ \\
\hline 0.4 & $5.23 \times 10^{-4}$ & $5.34 \times 10^{-5}$ & $7.47 \times 10^{-5}$ \\
\hline 0.5 & $2.15 \times 10^{-4}$ & $9.23 \times 10^{-1}$ & $4.04 \times 10^{-5}$ \\
\hline 0.6 & $1.05 \times 10^{-4}$ & $3.48 \times 10^{-6}$ & $1.12 \times 10^{-5}$ \\
\hline 0.7 & $4.18 \times 10^{-5}$ & $2.02 \times 10^{-6}$ & $5.93 \times 10^{-6}$ \\
\hline 0.8 & $1.88 \times 10^{-5}$ & $2.17 \times 10^{-1}$ & $2.73 \times 10^{-6}$ \\
\hline 0.9 & $7.35 \times 10^{-6}$ & $2.60 \times 10^{-1}$ & $1.24 \times 10^{-6}$ \\
\hline 1.0 & $3.17 \times 10^{-6}$ & $6.88 \times 10^{-8}$ & $3.90 \times 10^{-1}$ \\
\hline 1.1 & $1.22 \times 10^{-6}$ & $2.23 \times 10^{-8}$ & $1.76 \times 10^{-1}$ \\
\hline 1.2 & $5.12 \times 10^{-1}$ & $1.72 \times 10^{-8}$ & $7.48 \times 10^{-8}$ \\
\hline 1.3 & $1.96 \times 10^{-1}$ & $1.84 \times 10^{-9}$ & $3.18 \times 10^{-8}$ \\
\hline 1.4 & $8.05 \times 10^{-8}$ & $1.69 \times 10^{-9}$ & $1.05 \times 10^{-8}$ \\
\hline 1.5 & $3.09 \times 10^{-8}$ & $1.07 \times 10^{-9}$ & $4.46 \times 10^{-9}$ \\
\hline 1.6 & $1.25 \times 10^{-8}$ & $1.26 \times 10^{-11}$ & $1.82 \times 10^{-9}$ \\
\hline 1.7 & $5.39 \times 10^{-9}$ & $1.13 \times 10^{-10}$ & $7.50 \times 10^{-10}$ \\
\hline 1.8 & $2.22 \times 10^{-9}$ & $6.43 \times 10^{-11}$ & $2.54 \times 10^{-10}$ \\
\hline 1.9 & $1.60 \times 10^{-9}$ & $3.30 \times 10^{-12}$ & $1.04 \times 10^{-10}$ \\
\hline 2.0 & $1.06 \times 10^{-9}$ & $7.11 \times 10^{-12}$ & $4.16 \times 10^{-11}$ \\
\hline
\end{tabular}




\section{CONCLUSION AND ACKNOWLEDGEMENT}

It is evident from the above tables that our proposed methods are indeed accurate, and can handle stiff equations. Also in terms of stability analysis, the methods are A-stables and the scheme have also been shown to be of good order.

The authors will like to thank Dr. J. P. CHOLLOM for introducing them to the formation of hybrid block method through Multi-step Collocation and the use of MAPLE and MATLAB software.

\section{REFERENCES}

Dahlquist, G. (1963). A special stability problem for linear Multistep methods. BIT3 27-43.

Lambert, J. D. (1991). Numerical methods for ordinary differential Systems (Wiley) . p220.

Yakubu, D.G. New block implicit Runge-Kuta Collocation methods for solving stiff differential equations.Mathematical sciences programme, Abubakar Tafawa Balewa University, Bauchi, Nigeria.
England, R. and Mattheij, R. M. (1985). Sequential step control for Integration of two-point boundary value problems, Report 8541, Department of Mathematics, Catholic University, Toemooiveld, 6255

Ed Nijmegen, The Netherlands.

Onumanyi, P. Awoyemi, D. O. Jator, S. N. and Sirisena, U. W. (1994). New linear multi-step methods with continuous coefficients for first order initial value problems, J. Nig. Math. Soc. 13, 37-51.

Skwame, Y.(2005). Construction of Simpson-Type Hybrid Block methods for Stiff ordinary differential equations. M. Sc. Project. (Unpublished). University of Jos, Jos, Nigeria.

Chollon, J. P. A Study of block hybrid Adams methods with link To two step Runge-Kutta methods for first order ordinary differential Equations. Ph. D. Thesis. (2004). University of Jos, Jos, Nigeria.

Nakashima, M. (1992). Variable Coefficients A-Stable Explicit Runge-Kutta Methods. Proceedings of the Fifth International Conference on Scientific Computing. University of Benin, Nigeria. PP: 138-143. 